\title{
Análise eletromiográfica de músculos do abdome e reto femoral em exercícios abdominais com e sem superfície instável
}

http://dx.doi.org/10.11606/1807-5509201800020171

\author{
Frederico Balbino LIZARDO* \\ Gilmar da Cunha SOUSA* \\ Daniela Cristina de Oliveira SILVA* \\ Roberto Bernardino JÚNIOR* \\ Jacqueline Pereira Moraes RESENDE*** \\ Dioniz Bezerra da SILVA** \\ Fausto BÉRZIN*** \\ Delaine Rodrigues BIGATON***
}

\author{
* Universidade \\ Federal de \\ Uberlândia, \\ Uberlândia, MG, \\ Brasil. \\ ** Universidade \\ Presidente Antônio \\ Carlos, Uberlândia, \\ MG, Brasil. \\ ${ }^{* * \star}$ Faculdade \\ de Odontologia \\ de Piracicaba, \\ Universidade Estadual \\ de Campinas, \\ Piracicaba, SP, Brasil.
}

\section{Resumo}

Os exercícios abdominais são realizados visando a prevenção e/ou reabilitação de dores na região lombar, a melhoria do rendimento atlético, aumento da resistência e força do tronco durante o desempenho das atividades da vida diária e pela estética. Objetivou-se analisar e comparar a atividade eletromiográfica dos músculos rectus abdominis parte superior (RAS) e parte inferior (RAI), obliquus externus abdominis (OE), obliquus internus abdominis (OI) e rectus femoris (RF) durante o exercicio abdominal tradicional com e sem a utilização do bosu e bola de ginástica. A amostra foi composta por dez voluntários do gênero masculino, fisicamente ativos e sem distúrbio neuromuscular. A coleta de dados foi realizada utilizandose eletrodos de superfície diferenciais simples, com ganho de 20 vezes, e registrada por meio de um eletromiógrafo computadorizado. 0 sinal eletromiográfico foi quantificado pela Raiz Quadrada da Média (Root Mean Square [RMS]) e normalizado (RMSn) pela Contração Isométrica Voluntária Máxima. Os dados foram submetidos à análise estatística paramétrica, empregando-se teste de análise de variância de medidas repetidas (Anova). Os resultados demonstraram que atividade do músculo RAS no exercício com bosu foi significativamente maior em relação ao tradicional $(p<0.05)$, todavia, não foram encontradas diferenças significativas ( $p>0.05$ ) para músculos $R A I, O E, O I$ e $R F$, entre todos exercícios abdominais (tradicional, bosu e bola de ginástica). Conclui-se que a utilização do bosu no exercício abdominal pode ser um fator desejável e necessário em estágios específicos de programas de reabilitação e/ou treinamento físico, principalmente quando objetiva-se aumentar o recrutamento da parte superior do músculo rectus abdominis (RAS).

Palavras- chave: Cinesiologia; Rectus Abdominis; Bosu; Bola de Ginástica.

\section{Introdução}

Os exercícios abdominais são realizados visando à prevenção e/ou reabilitação de dores na região lombar (lombalgia), a melhoria do rendimento atlético, aumento da resistência e força do tronco durante o desempenho das atividades da vida diária e pela crescente busca da estética que é divulgada pela nossa sociedade ${ }^{1-3}$.
Nos exercícios abdominais deve ser priorizado o recrutamento dos músculos rectus abdominis (RA), obliquus externus abdominis (OE), obliquus internus abdominis (OI) e transversus abdominis (TA) de forma a minimizar a atividade do músculo rectus femoris $(\mathrm{RF})^{4}$. Segundo SundsTRup et al..$^{5}$ o aumento da atividade do músculo RF nos exercícios 
abdominais provoca maior tração e força de cisalhamento na regiâo lombar da coluna vertebral.

Em função disso, investigaçóes têm sido conduzidas a fim de determinar a maneira mais eficiente e correta de se realizar exercícios abdominais, principalmente por meio da eletromiografia de superfície $^{6-10}$. Esta, segundo Basmajian e De Luca ${ }^{11}$, é o estudo da função muscular a partir da averiguação do sinal elétrico que emana do músculo.

Vários aparelhos e instrumentos podem ser utilizados na execução dos exercícios abdominais, dentre eles destacam-se as superfícies instáveis que estão disponíveis na área de fitness e reabilitação física. Um dos instrumentos com superfície instável mais conhecido atualmente é a bola de ginástica, também denominada de bola suíça ou physioball ${ }^{2}$.

A bola de ginástica é um instrumento comumente utilizado por treinadores e outros profissionais da saúde em vários tipos de aulas nas academias, clubes e, principalmente, em reabilitação fisioterápica, já que esta aumenta o nível de dificuldade dos exercícios, altera as exigências proprioceptivas e melhora o controle motor dos músculos do tronco importantes para equilíbrio e estabilidade ${ }^{5,12,13}$.

O bosu balance trainer também é um instrumento de superfície instável que possui uma sólida plataforma de base com uma redoma de borracha inflável, semelhante a uma bola de ginástica partida ao meio ${ }^{2,14}$. O bosu balance trainer tornou-se bastante popular nas academias de ginástica e clínicas de versátil que pode ser utilizado com a plataforma apoiada no chão ou virada para cima em diferentes tipos de exercícios ${ }^{14}$.

A estabilidade durante o exercício pode ficar comprometida caso a base de suporte (superfície instável) não possua uma boa área de contato com o solo ${ }^{15}$, desta forma, o tamanho da área de contato da base de suporte com o solo é um fator a ser terapia física por ser considerado um instrumento

considerado para se determinar a estabilidade exigida no exercício. Alguns autores analisaram a atividade eletromiográfica dos músculos do tronco em exercícios abdominais e de estabilização com e sem utilização de diferentes superfícies instáveis ${ }^{3,5,16-19}$. Imar et al. ${ }^{20}$ demonstraram maior atividade do músculo OE no exercício abdominal com bosu em comparação com o tradicional.

Desta forma, devido a popularidade das superfícies instáveis e sua aplicação na área de reabilitação e treinamento, em especial a bola de ginástica e o bosu, torna-se importante verificar os efeitos da utilização destes equipamentos no exercício abdominal para preencher lacunas existentes na literatura e responder dúvidas, tais como: (1) A utilização da bola de ginástica produzirá maior ativação dos músculos abdominais em comparação com bosu? (2) Qual superfície instável produzirá maior recrutamento dos músculos abdominais em comparação com exercício tradicional?

A compreensão dessas questôes é importante para proporcionar uma base teórica para o direcionamento de programas de prevenção, reabilitação e treinamento esportivo que objetivam o desenvolvimento e fortalecimento dos músculos abdominais.

O objetivo deste estudo foi analisar e comparar a atividade eletromiográfica dos músculos abdominais (RA, OE, OI) e rectus femoris durante a execução do exercício abdominal tradicional com e sem a utilização do bosu e bola de ginástica. A hipótese é que a utilização de superfície instável no exercício abdominal proporcionará aumento na atividade eletromiográfica dos músculos abdominais, além disso, acredita-se que a utilização da bola de ginástica como superfície instável produzirá maior recrutamento dos músculos abdominais em comparação ao bosu, por apresentar menor base de suporte e área de contato com o solo.

\section{População e amostra}

Trata-se de um estudo de caráter experimentalquantitativo, aprovado pelo Comitê de Ética em Pesquisa (número 005755) da Universidade Federal de Uberlândia (UFU) e desenvolvido no Laboratório de Eletromiografia Cinesiológica (LABEC) da UFU. gênero masculino com idade entre $26.4 \pm 6.3$ anos, massa corporal $75.6 \pm 9.8 \mathrm{~kg}$ e estatura $174.1 \pm 6.1 \mathrm{~cm}$. Foram selecionados apenas indivíduos sem histórico de dores na regiáo lombar e/ou abdominal e distúrbio neuromuscular ou articular. Todos voluntários eram
A amostra foi composta por dez voluntários do 
fisicamente ativos de acordo com classificação do questionário internacional de atividade física (IPAQ versão curta) e tinham experiência em treinamento resistido no período mínimo de seis meses anterior a este estudo, especialmente em exercícios específicos para os músculos da regiāo abdominal, como exercício tradicional (crunch) $)^{3,4}$.

O tamanho da amostra (n) foi determinado por meio do cálculo amostral com base no parâmetro eletromiográfico RMS da parte superior do músculo rectus abdominis (RAS), obtido em um estudo piloto com três voluntários. O cálculo amostral foi realizado utilizando-se o aplicativo GPower 3.1, power de $80 \%$ e alpha $=0,05$. Este cálculo forneceu uma amostra de tamanho mínimo de $\mathrm{n}=8$ para este estudo.

\section{Procedimento experimental}

A coleta de dados foi dividida em dois dias distintos. No primeiro, os voluntários passaram por avaliação física (altura e massa corporal), responderam o Questionário Internacional de Atividade Física - Versão Curta e realizaram a familiarização dos exercícios e da contração isométrica voluntária máxima (CIVM). A coleta dos dados eletromiográficos ocorreu uma semana depois, no qual foram realizadas duas CIVM de cinco segundos para cada músculo com período de descanso de três minutos $^{21}$, para posterior normalização dos dados. Os testes de CIVM dos músculos RA, OE, OI, e RF estão descritos a seguir:

1. Músculo RA: os sujeitos ficaram deitados no chão sobre um colchonete em posiçáo de decúbito dorsal com quadris e joelhos flexionados a $90^{\circ}$, os pés fixos no chão e com uma flexão parcial do tronco até que as escápulas fossem elevadas do solo (supraabdominal). Foram instruídos na tentativa de realizar uma flexão da parte superior do tronco no plano sagital com resistência manual do pesquisador na região do ombro e no sentido da extensão do tronco ${ }^{3,18}$.

2. Músculos OE e OI: os sujeitos ficaram deitados no chão sobre um colchonete em posição de decúbito dorsal com quadris e joelhos flexionados a $90^{\circ}$, os pés fixos no chão e o tronco em flexão e rotação pra direita ou esquerda. Foram instruídos a manter a flexão do tronco com rotação para a direita ou esquerda, sendo aplicada pelos pesquisadores uma resistência manual no ombro no sentido contrário ao movimento, ou seja, na direção de extensão e rotação contra-lateral do tronco ${ }^{3,18}$.

3. Músculo RF - os sujeitos ficaram em posição sentada com quadril e joelhos flexionados a $90^{\circ}$. Foram instruídos na tentativa de realizar uma extensão da perna contra uma resistência manual do pesquisador na parte distal da perna no sentido contrário ao movimento ${ }^{3}$.

Após o término das CIVM, foram utilizados dez minutos de intervalo e, posteriormente, os sujeitos realizaram todos os exercícios abdominais. A ordem de execuçáo destes foi aleatória por meio de sorteio. Os dados eletromiográficos foram coletados durante uma série de cinco repetiçóes para cada exercício abdominal, os quais foram realizados num ritmo cadenciado de aproximadamente dois segundos para a fase concêntrica e dois segundos para a fase excêntrica ${ }^{1,3}$.

Foi utilizado um metrônomo (fixado com um batimento por segundo) para ajudar e garantir a cadência adequada durante os exercícios abdominais. Para evitar o efeito da fadiga muscular como fator limitante foram utilizados dois minutos de intervalo de descanso entre cada exercício ${ }^{3,4}$.

\section{Exercícios executados}

Foi construído e utilizado um aparelho com duas barras verticais e uma barra horizontal regulável de canos de Policloreto de vinila (PVC), destinado a limitar a fase concêntrica do movimento e adaptado de acordo com Hildenbrand e Noble ${ }^{12}$. O sujeito realizou o exercício até tocar o tórax na barra horizontal, que foi regulada no ponto que o voluntário realizava a flexão parcial do tronco no qual apenas as escápulas eram levantadas do chão. Os exercícios estão ilustrados na FIGURA 1.

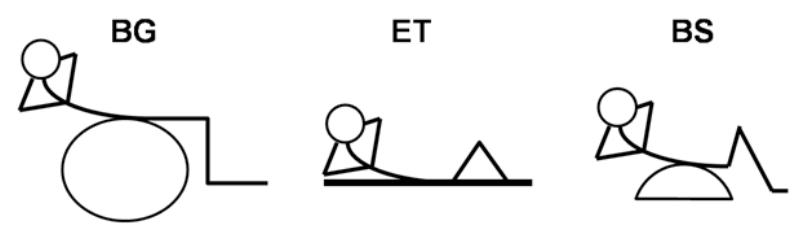

FIGURA 1 - Desenho esquemático dos diferentes exercícios abdominais. Bola de ginástica (BG), Exercício tradicional (ET) e Bosu (BS). 


\section{Posiçáo inicial do exercício tradicional}

Os sujeitos permaneceram em decúbito dorsal sobre um colchonete com quadris e joelhos flexionados a $90^{\circ}$ e os pés fixos no solo.

\section{Posiçáo inicial do exercício com bosu}

Os sujeitos permaneceram em decúbito dorsal com a regiáo lombar apoiada no bosu, quadris e joelhos flexionados a $90^{\circ}$ e os pés apoiados no solo.

\section{Posiçâo inicial do exercício com bola de ginástica}

Os sujeitos permaneceram em decúbito dorsal com a região lombar apoiada na bola de ginástica, os quadris em posição neutra, joelhos flexionados a $90^{\circ}$ e os pés apoiados no solo.

\section{Posição final dos exercícios abdominais}

Os voluntários foram instruídos a realizar uma flexão do tronco no plano sagital até encostar o tórax na barra horizontal do aparelho de PVC, com os polegares colocados sobre as orelhas, as mãos relaxadas contra a cabeça e os cotovelos afastados lateralmente e, em seguida, retornaram à posição inicial.

O tamanho da bola de ginástica (Gym Ball, Adidas Performance - FIT4 STORE, Campinas, SP, Brasil) foi selecionado de acordo com a altura do sujeito, aqueles com altura entre 150 e 170 centímetros (cm) foi utilizado uma bola de $55 \mathrm{~cm}$ diâmetro e os que apresentaram altura superior a $170 \mathrm{~cm}$ foi utilizado uma bola de $65 \mathrm{~cm}^{5}$. A bola de ginástica foi inflada de acordo com o peso do voluntário, o qual ficou sentado no centro da bola com o tronco ereto, pés unidos em contato com chão, quadris e joelhos flexionados aproximadamente em $90^{\circ} \mathrm{e}$ coxas paralelas ao solo ${ }^{3}$.

O equipamento bosu (Bosu Balance - ISP Dyna, Campinas, SP, Brasil) é versátil e resistente, suporta até $200 \mathrm{~kg}$, possui $55 \mathrm{~cm}$ diâmetro e combina uma sólida plataforma de base com uma cúpula azul de borracha inflável. O dispositivo bosu foi inflado até uma altura recomendada de aproximadamente 25.4 $\mathrm{cm}$, no qual a plataforma de base ficava apoiada no chão e a cúpula azul de borracha virada para cima ${ }^{14}$.

\section{Eletromiografia}

Os registros eletromiográficos foram obtidos utilizando-se um eletromiógrafo computadorizado da
Myosystembr1 P84/DATAHOMINIS Tecnologia ${ }^{\circledR}$ (Uberlândia, MG, Brasil) projetado de acordo com normas internacionais, o qual possui impedância de entrada de 1015 Ohms, conversor analógico/ digital com resolução de 16 bits, filtros Butterworth, filtro passa-alta de $20 \mathrm{~Hz}$ e passa-baixa de $500 \mathrm{~Hz}$ e bateria recarregável integrada. $\mathrm{O}$ MyosystemBr1 P84 foi conectado a um laptop/notebook alimentado apenas por bateria. Os sinais eletromiográficos foram coletados e processados posteriormente usando um aplicativo de software Myosystem Brl (versão 3.5.6). A frequência de amostragem utilizada foi de $2000 \mathrm{~Hz}$ por canal durante toda a coleta.

Para captação dos sinais eletromiográficos foram utilizados eletrodos de superfície diferenciais simples (DataHominis Tecnologia Ltda., Uberlândia, MG, Brasil), com ganho de 20 vezes e razão de rejeição em modo comum de $92 \mathrm{~dB}$ a $60 \mathrm{~Hz}$, compostos por duas barras retangulares paralelas de prata pura $(10 \mathrm{~mm}$ de comprimento x $1 \mathrm{~mm}$ de largura) e distanciadas 10 $\mathrm{mm}$ entre si. A preparação dos voluntários consistiu em tricotomia e limpeza da pele com álcool $70 \%$.

Os eletrodos de superfície foram colocados nos músculos do antímero direito ${ }^{3,4,22}$, com sua orientação paralela e as barras de detecção do sinal perpendicular ao sentido das fibras musculares ${ }^{23}$. Para a parte superior do músculo rectus abdominis (RAS), o eletrodo foi alinhado verticalmente e fixado no centro do ventre muscular no ponto médio entre o processo xifoide do osso esterno e cicatriz umbilical (mas não na intersecção tendínea) e $3 \mathrm{~cm}$ lateral à linha mediana do corpo $^{3}$; o eletrodo da parte inferior do músculo rectus abdominis (RAI) foi colocado no centro do ventre muscular no ponto médio entre cicatriz umbilical e sínfise púbica e $3 \mathrm{~cm}$ lateral à linha mediana ${ }^{3}$.

O eletrodo no músculo OE foi colocado superiormente à espinha iliaca ântero-superior (EIAS) do osso do quadril, aproximadamente a 15 centímetros laterais da cicatriz umbilical ${ }^{3,24}$. O eletrodo do músculo OI foi alinhado horizontalmente e colocado dois centímetros inferior e medial à EIAS do osso do quadril ${ }^{3,24}$, localizado no interior de um triângulo delimitado pelo ligamento inguinal, borda lateral da bainha do reto e uma linha que conecta ambas EIAS, onde apenas a aponeurose do OE e não suas fibras musculares encobre $\mathrm{o} \mathrm{OI}^{3}$.

Para o músculo RF, o eletrodo foi colocado no ponto médio $(50 \%)$ da linha que se estende entre a espinha ilíaca ântero-superior do osso do quadril até a borda proximal da patela ${ }^{3}$. Foi utilizado um eletrodo de referência (Bio-logic Systems - SP Médica, Científica e Comercial Ltda., São Paulo, SP, Brasil) fixado na 
pele sobre a espinha ilíaca ântero-superior do osso do quadril esquerdo ${ }^{25,26}$.

As análises dos sinais eletromiográficos foram realizadas individualmente para averiguação do registro e rotina do sinal, sendo considerados somente os sinais que não apresentassem interferência de qualquer natureza. Para isso, todas as coletas do sinal eletromiográfico pertencentes ao procedimento experimental foram precedidas de análise em tempo real do espectro de frequência, o qual permite observar eventuais interferências que possam estar presentes nas coletas ${ }^{27}$.

\section{Análise dos dados}

Os sinais eletromiográficos obtidos durante as CIVM e todos os exercícios abdominais foram analisados e quantificados no domínio do tempo utilizando o parâmetro Root Mean Square (RMS). Os valores de RMS foram calculados a partir de uma janela de tempo de três segundos centrais na CIVM e, nos exercícios abdominais, o valor médio de RMS foi calculado a partir de uma janela móvel de um segundo durante três repetiçóes de cinco realizadas, sendo que a primeira e última contração foram excluídas. $\mathrm{O}$ valor máximo da CIVM foi utilizado para a normalização dos dados e os valores de coeficientes de correlação intraclasse (ICC) de RMS nos testes de CIVM foram
0.943 (RAS), 0.938 (RAI), 0.955 (OE), 0.965 (OI) e 0.708 (RF). Além disso, os valores médios de ICC do sinal eletromiográfico durante os exercícios abdominais foram 0.986 (RAS), 0.984 (RAI), 0.919 (OE), 0.977 (OI) e 0.721 (RF).

\section{Análise estatística}

A análise estatística dos dados foi realizada utilizando-se o programa computadorizado GraphPad Prism (versão 3.0 - Graphpad Software, Inc). Utilizou-se o teste Kolmogorov-Smirnov para avaliar a normalidade dos dados e, posteriormente, testes paramétricos foram empregados em todas as análises. A análise de variância de medidas repetidas (Anova) foi utilizada para comparaçáo das médias dos valores de RMS normalizado (RMSn) do mesmo músculo em diferentes exercícios. Em todas estas análises foi realizado o teste de comparaçóes múltiplas de Tukey para verificar onde havia diferença e o nível de significância foi estabelecido em 5\% ( $<<0.05)$. Todos os resultados são apresentados na forma de média e desvio padrão e o tamanho do efeito (effect size [ES]) foi calculado utilizando a descrição de Cohen's para interpretação $(E S=0.2$ pequeno; $\mathrm{ES}=0.5$ médio e $\mathrm{ES}=0.8$ grande) .

\section{Resultados}

Os valores da atividade eletromiográfica (valores médios de RMSn) dos músculos RAS, RAI, $\mathrm{OE}, \mathrm{OI}$ e RF durante os exercícios abdominais (tradicional, bosu e bola de ginástica) estão demonstrados na TABELA 1 e FIGURA 2.

A atividade do músculo RAS no exercício com bosu foi significativamente maior em relação ao tradicional $(\mathrm{p}<0.05 ; \mathrm{ES}=0.79)$, entretanto,

não foram demonstradas diferenças significativas ( $p>0.05)$ no sinal eletromiográfico entre os exercícios da forma tradicional e bola de ginástica e os exercícios com bosu e bola de ginástica.

Em relação aos músculos RAI, OE, OI e RF, não foram encontradas diferenças significativas ( $\mathrm{p}>0.05$ ) dos valores médios de RMSn entre os distintos exercícios abdominais (tradicional, bosu e bola de ginástica).

TABELA 1 - Análise descritiva dos resultados com os valores médios do RMSn (expressos em \% CIVM) e desvio padrão dos músculos RAS, RAI, OE, OI e RF durante os diferentes exercícios abdominais (tradicional, bosu e bola de ginástica). Uberlândia-MG, 2015.

\begin{tabular}{lccccc}
\hline \multirow{2}{*}{ Exercícios } & \multicolumn{5}{c}{ Músculos } \\
\cline { 2 - 6 } \multicolumn{1}{c}{ RAS } & RAI & OE & OI & RF \\
\hline Tradicional & $58.77 \pm 7.9$ & $58.56 \pm 12.3$ & $40.84 \pm 12.1$ & $41.36 \pm 13.4$ & $9.30 \pm 8.9$ \\
Bosu & $73.03 \pm 24.3$ & $66.54 \pm 17.0$ & $61.51 \pm 27.8$ & $50.64 \pm 18.2$ & $7.67 \pm 6.8$ \\
Bola ginástica & $65.54 \pm 23.1$ & $63.03 \pm 16.3$ & $60.44 \pm 21.6$ & $42.93 \pm 18.9$ & $9.02 \pm 6.1$ \\
\hline
\end{tabular}


*: Diferença

significativa em

relação ao exercício tradicional.

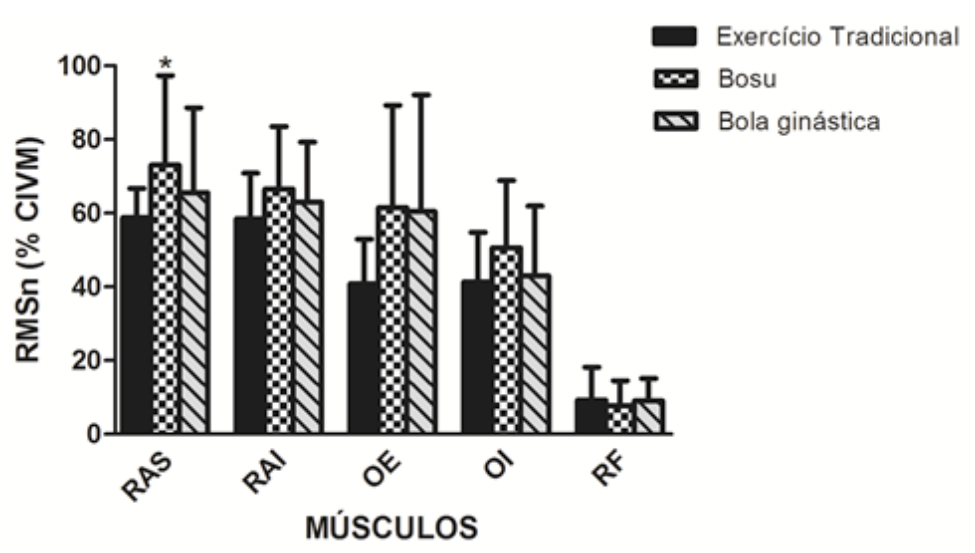

FIGURA 2 - Comparação dos valores médios de RMSn dos músculos RAS, RAI, OE, OI e RF durante o exercício abdominal tradicional (ET), bosu (BS) e bola de ginástica (BG). As barras representam a média e o desvio padrão.

\section{Discussão}

Os resultados da presente pesquisa denotam que no exercício abdominal com bosu foi verificado maior atividade eletromiográfica do músculo RAS em comparação ao exercício tradicional, todavia, não foram demonstradas diferenças significativas no sinal eletromiográfico dos músculos RAI, OE, OI e RF durante todos exercícios abdominais com (bosu e bola de ginástica) e sem superfície instável (tradicional).

Os músculos RAS e RAI apresentaram atividade eletromiográfica similar nos diferentes exercícios, com exceção do músculo RAS no exercício abdominal com bosu em comparação com o tradicional. Segundo Moore, Dalley e Agur ${ }^{28}$, o músculo RA é um forte flexor do tronco, aproximando a margem costal anterior do osso púbis, sendo assim, em todos exercícios analisados no presente estudo, os voluntários tiveram que encostar o tórax na barra horizontal ao mesmo tempo em que removessem por completo as escápulas da superfície de apoio, realizando um movimento puro de flexão do tronco, com a velocidade do movimento limitada e controlada pelo metrônomo, situação que explica o padrão de recrutamento similar do músculo rectus abdominis nos diferentes exercícios.

Entretanto, como citado anteriormente, a atividade do músculo RAS no exercício com bosu foi significativamente maior em relação ao tradicional $(\mathrm{p}<$ $0.05)$. Essa diferença de ativação pode ser justificada pela relação comprimento-tensão, pois, segundo BANKOFF ${ }^{29}$, essa relaçáo estabelece que o comprimento muscular mais indicado para gerar tensão é levemente superior ao comprimento de repouso. Quando o músculo ativado é alongado antes do encurtamento, sua performance é aumentada durante a fase isotônica-concêntrica ${ }^{30}$.
No exercício com bosu, a base plana ficou apoiada firmemente no solo e a superfície convexa e macia em contato com a região lombar do voluntário, sendo assim, mesmo os voluntários realizando a flexão do tronco em ambos os exercícios (bosu e tradicional), o ângulo do espaço percorrido pelo tronco durante os exercícios foi maior com o bosu, provocando maior torque de extensão na coluna e maior préestiramento do músculo RAS em comparação com o tradicional, fator que pode explicar a diferença de ativação entre ambos exercícios.

Os exercícios abdominais realizados no presente trabalho com bosu e bola de ginástica podem ser considerados exercícios instáveis, porém, não foram registradas diferenças significativas na atividade dos músculos abdominais e RF entre ambos os exercícios. Mesmo com ausência de medidas específicas relacionadas com o tamanho da área de contato da superfície instável com o solo, nota-se, de forma subjetiva, que no exercício com bosu a plataforma que possui uma base plana fica apoiada firmemente no solo, enquanto que a bola de ginástica possui uma base de contato com o solo convexa, possuindo menor base de suporte e menor área de contato. Todavia, este fator não foi preponderante para produzir diferenças no sinal eletromiográfico dos músculos abdominais e RF entre ambos exercícios.

De acordo com WeINECK ${ }^{31}$, a contração bilateral do músculo $\mathrm{OE}$ e OI produz flexáo do tronco, atuando em sinergia com músculo RA, enquanto que a contração unilateral resulta em inclinaçáo do tronco para o mesmo lado ou rotaçáo do tronco para o lado oposto. Ainda, segundo WeINecK ${ }^{31}$, quando o músculo OI realiza 
uma contração bilateral ele participa da flexão do tronco e na contração unilateral atua na inclinaçáo e rotação do tronco para o mesmo lado. Na presente pesquisa foram realizados exercícios abdominais com apenas movimentos de flexáo do tronco sem rotação, entretanto, foi observado uma ativação similar dos músculos oblíquos durante os diferentes exercícios, corroborando as afirmações do autor supracitado sobre a ação sinérgica destes músculos na flexão do tronco.

Segundo CAMPos e $\mathrm{NeTO}^{15}$, a forma da bola permite o aparecimento de um torque de extensão para a coluna que deve ser contraposto pelos músculos abdominais, e ao mesmo tempo, proporciona instabilidade láterolateral, produzindo um torque de rotação da coluna. Sendo assim, os músculos oblíquos são importantes para contrapor o torque de extensão e principalmente o torque de rotação da coluna, fatores que proporcionaram maior recrutamento desta musculatura com superfícies instáveis. No entanto, os resultados da presente pesquisa não demonstraram diferenças na ativaçáo dos músculos oblíquos durante os diferentes exercícios, contrastando com estas afirmaçóes.

IMAI et al. ${ }^{20}$ compararam a atividade elétrica bilateral dos músculos RA, OE e TA durante a realização do exercício tradicional com e sem bosu, os quais demonstraram atividade significativamente maior no OE e menor no TA com a utilizaçáo do bosu em comparação com o tradicional. $\mathrm{Na}$ presente pesquisa foi observado que apenas a atividade do músculo RAS no exercício com bosu foi significativamente maior, contrastando com as afirmaçóes dos autores supracitados.

Deve-se ressaltar que IMAi et al. ${ }^{20}$ analisaram o exercício no bosu utilizando os pés levantados, cruzados e sem contato com o chão, enquanto que neste trabalho, o exercício foi realizado com os pés em contato com o solo. Esta alteração pode explicar as diferenças nos resultados, ou seja, com os pés fixos no chão o indivíduo teve maior equilíbrio e conseguiu ter um maior foco na flexão do tronco, proporcionando maior recrutamento do RAS. Por outro lado, com os pés sem contato com o solo, acredita-se que foi necessário maior controle neuromuscular para manutençáo do equilíbrio devido a maior instabilidade látero-lateral, provocando diferenças maiores no padrão de ativação do $\mathrm{OE}$.

Não foram demonstradas diferenças significativas na atividade elétrica de todos os músculos abdominais no exercício com bola de ginástica em comparação com o tradicional (FIGURA 2). Esses achados estáo de acordo com os resultados de HILDENBRAND e NOBLE ${ }^{12}$ e LizARDo et al. ${ }^{13}$, entretanto, Vera-GarCia, GrenIER
E McGILL ${ }^{32}$ demonstraram diferenças significativas entres estes exercícios, no qual a utilização da bola produziu o dobro da atividade elétrica para o músculo RA e quatro vezes mais para o OE. No estudo de Vera-Garcia, Grenier e McGill ${ }^{32}$ foram utilizadas somente contraçóes isométricas e os autores não se preocuparam com o nível de atividade física e de experiência dos voluntários em relação ao exercício abdominal, fatores que podem explicar estas variaçóes.

Monfort-PAÑEgo et al. ${ }^{33}$ recomendaram realizar exercícios de flexão da coluna vertebral com joelhos e quadris flexionados para neutralizar a lordose lombar, reduzir a tensão no músculo psoas maior e diminuir o envolvimento da musculatura flexora da coxa. Segundo Sundstrup et al. ${ }^{5}$, o aumento da atividade dos músculos RF ou iliopsoas nos exercícios abdominais provocará maior tração e força de cisalhamento na regiáo lombar da coluna, não sendo indicado para pessoas com dor lombar ou instabilidade lombar em geral.

Os exercícios abdominais da forma tradicional e com bosu foram realizados com flexão da coxa, enquanto que o exercício com bola foi executado com a coxa na posição neutra (paralela ao solo). Apesar dessas alteraçôes e do curto tempo de familiarizaçáo com o exercício nas superfícies instáveis, o que poderia aumentar o nível de dificuldade e proporcionar maior ativação elétrica dos músculos estabilizadores da pelve como o $\mathrm{RF}^{12}$, não ocorreram diferenças significativas na ativação deste músculo nos diferentes exercícios abdominais, sendo que todos estes foram eficazes na redução da atividade do músculo flexor da coxa em comparação com os músculos abdominais, priorizando o fortalecimento abdominal e minimizando o fator prejudicial de maior sobrecarga na regiáo lombar da coluna vertebral.

No presente estudo foi utilizado um grupo de voluntários do gênero masculino, relativamente jovens, saudáveis, fisicamente ativos e sem histórico de dores na região lombar, portanto, os resultados devem ser aplicados com precauçóes para diferentes populaçóes, tornando-se um fator limitante deste trabalho. Futuros estudos podem realizar comparaçôes da atividade eletromiográfica em grupos distintos como idosos, sedentários ou sujeitos com lombalgia. Além disso, a ausência de medidas específicas relacionadas com o tamanho da área de contato da superfície instável com o solo pode ser considerada outro fator de limitação.

Conclui-se que a atividade eletromiográfica do músculo RAS foi significativamente maior no exercício abdominal com bosu em comparação com exercício tradicional, portanto, a utilização 
deste equipamento instável no exercício abdominal pode ser um fator desejável e necessário em estágios específicos de programas de reabilitação e/ou treinamento físico.

\section{Abstract \\ Electromyography of the abdominal muscles and rectus femoris in abdominal exercises with and without the unstable surfaces}

The abdominal exercises are performed to the preventing and/or rehabilitation of lower back pain, improved athletic performance, increased strength and resistance of the trunk during the performance of activities of daily living and aesthetics. The objective was to analyze and compare the electromyographic activity of the upper (URA) and lower rectus abdominis (LRA), external oblique (EO), internal oblique (IO) and rectus femoris (RF) during traditional abdominal exercise with and without using the bosu and gymnastics ball. The sample was composed of 10 male volunteers, active physically and without neuromuscular disorder. Data collection was performed using a single differential surface electrodes, with a gain of 20 times, and recorded by a computerized electromyography. The electromyographic signal was quantified by the Root Mean Square (RMS) and normalized (RMSn) by maximal voluntary isometric contraction. The data were subjected to parametric statistical analysis, using the analysis of variance (ANOVA) for repeated measures. The results showed that the URA muscle activity in the exercise with bosu was significantly higher compared to the traditional $(p<0.05)$, however, for the LRA, EO, IO and RF muscles, there were no significant differences ( $p>0.05$ ) among all abdominal exercises (traditional, bosu and gymnastics ball). We conclude that the use of the bosu in the abdominal exercises can be a necessary and desirable factor in specific stages of rehabilitation programs and / or physical training, mainly to increase the recruitment of the upper (URA) rectus abdominis muscle.

KeYwords: Kinesiology; Rectus Abdominis; Bosu; Gymnastics Ball.

\section{Referências}

1. Lizardo FB, Sousa GC, Facury Neto MA, Marques KV, Bernardino Júnior R, Silva DCO, et al. Comparative electromyographic analysis of the rectus abdominis and rectus femoris muscles in abdominal exercises with or without the ab swing device. Biosci J. 2009;25(3):92-103.

2. Behm DG, Drinkwater EJ, Willardson JM, Cowley PM. Canadian Society for Exercise Physiology position stand: the use of instability to train the core in athletic and nonathletic conditioning. Appl Physiol Nutr Metab. 2010;35(1):109-12.

3. Escamilla RF, Lewis C, Bell D, Bramblet G, Daffron J, Lambert S, et al. Core muscle activation during Swiss ball and traditional abdominal exercises. J Orthop Sports Phys Ther. 2010;40(5):265-76.

4. Youdas JW, Guck BR, Hebrink RC, Rugotzke JD, Madson TJ, Hollman JH. An electromyographic analysis of the Ab-Slide exercise, abdominal crunch, supine double leg thrust, and side bridge in healthy young adults: implications for rehabilitation professionals. J Strength Cond Res. 2008;22(6):1939-46.

5. Sundstrup E, Jakobsen MD, Andersen CH, Jay K, Andersen LL. Swiss ball abdominal crunch with added elastic resistance is an effective alternative to training machines. Int J Sports Phys Ther. 2012;7(4):372-80.

6. Sullivan W, Gardin FA, Bellon CR, Leigh S. The effect of the traditional versus a modified bent-knee sit-up on abdominal and hip flexor muscle electromyographic activity. J Strength Cond Res. 2015;29(12):3472-9.

7. Saeterbakken AH, Andersen V, Jansson J, Kvellestad AC, Fimland MS. Effects of bosu ball(s) during sit-ups with body weight and added resistance on core muscle activation. J Strength Cond Res. 2014;28(12):3515-22.

8. Burden AM, Redmond CG. Abdominal and hip flexor muscle activity during 2 minutes of sit-ups and curl-ups. J Strength Cond Res. 2013;27(8):2119-28.

9. López-Valenciano A, Biviá-Roig G, Lisón JF, Vera-Garcia FJ. Estudio electromiográfico de ejercicios de flexión del tronco sobre banco inclinado. Rev Int Med Cienc Act Fís Deporte. 2013;13(52):657-71.

10. Rutkowska-Kucharska A, Szpala A. Electromyographic muscle activity in curl-up exercises with different positions of upper and lower extremities. J Strength Cond Res. 2010;24(11):3133-9. 
11. Basmajian JV, De Luca CJ. Muscles alive: their function revealed by electromyography. 5a ed. Baltimore: Williams and Wilkins; 1985.

12. Hildenbrand K, Noble L. Abdominal muscle activity while performing trunk-flexion exercises using the ab roller, abslide, fitball, and conventionally performed trunk curls. J Athl Train. 2004;39(1):37-43.

13. Lizardo FB, Sousa GC, Oliveira DCS, Marques KV, Bernardino Júnior R. Análise eletromiográfica da atividade elétrica dos músculos reto do abdome e reto femoral em exercícios abdominais com e sem bola de ginástica. Coleção Pesq Educ Fisica. 2007;6(1):87-94.

14. Laudner KG, Koschnitzky MM. Ankle muscle activation when using the Both Sides Utilized (BOSU) balance trainer. J Strength Cond Res. 2010;24(1):218-22.

15. Campos MA, Neto BC. Treinamento funcional resistido: para melhoria da capacidade funcional e reabilitação de lesões musculoesqueléticas. Rio de Janeiro: Revinter; 2008.

16. Schoffstall JE, Titcomb DA, Kilbourne BF. Electromyographic response of the abdominal musculature to varying abdominal exercises. J Strength Cond Res. 2010;24(12):3422-6.

17. Kang H, Jung J, Yu J. Comparison of trunk muscle activity during bridging exercises using a sling in patients with low back pain. J Sports Sci Med. 2012;11(3):510-5.

18. Byrne JM, Bishop NS, Caines AM, Crane KA, Feaver AM, Pearcey GEP. Effect of using a suspension training system on muscle activation during the performance of a front plank exercise. J Strength Cond Res. 2014;28(11):3049-55.

19. Vera-Garcia FJ, Barbado D, Moya M. Trunk stabilization exercises for healthy individuals. Rev Bras Cineantropom Desempenho Hum. 2014;16(2):200-11.

20. Imai A, Kaneoka K, Okubo Y, Shiina I, Tatsumura M, Izumi S, et al. Trunk muscle activity during lumbar stabilization exercises on both a stable and unstable surface. J Orthop Sports Phys Ther. 2010;40(6):369-75.

21. Brown LE. Treinamento de força. Barueri: Manole; 2008.

22. Hibbs AE, Thompson KG, French DN, Hodgson D, Spears IR. Peak and average rectified EMG measures: which method of data reduction should be used for assessing core training exercises? J Electromyogr Kinesiol. 2011;21(1):102-11.

23. De Luca CJ. The use of surface electromyography in biomechanics. J Appl Biomech. 1997;13(2):135-63.

24. García-Vaquero MP, Moreside JM, Brontons-Gil E, Peco-González N, Vera-Garcia FJ. Trunk muscle activation during stabilization exercises with single and double leg support. J Electromyogr Kinesiol. 2012;22(3):398-406.

25. Escamilla RF, Babb E, DeWitt R, Jew P, Kelleher P, Burnham T, et al. Electromyographic analysis of traditional and nontraditional abdominal exercises: implications for rehabilitation and training. Phys Ther. 2006;86(5):656-71.

26. Queiroz BC, Cagliari MF, Amorim CF, Sacco IC. Muscle activation during four pilates core stability exercises in quadruped position. Arch Phys Med Rehabil. 2010;91(1):86-92.

27. Aguiar AP. Análise eletromiográfica e do lactato sanguíneo em exercício resistido incremental [Dissertação]. Piracicaba: Faculdade de Ciências da Saúde, Universidade Metodista de Piracicaba; 2006.

28. Moore KL, Dalley AF, Agur AMR. Anatomia orientada para a clínica. 6a ed. Rio de Janeiro: Guanabara Koogan; 2011.

29. Bankoff ADP. Morfologia e cinesiologia: aplicada ao movimento humano. Rio de Janeiro: Guanabara Koogan; 2007.

30. Kubo K, Kanehisa H, Kawakami Y, Fukunaga T. Influence of static stretching on viscoelastic properties of human tendon structures in vivo. J Appl Physiol. 2009;90(2):520-7.

31. Weineck J. Anatomia aplicada ao esporte. 18a ed. Barueri: Manole; 2013.

32. Vera-Garcia FJ, Grenier SG, McGill SM. Abdominal muscle response during curl-ups on both stable and labile surfaces. Phys Ther. 2000;80(6):564-9.

33. Monfort-Pañego M, Vera-García FJ, Sánchez-Zuriaga D, Sarti-Martínez MA. Electromyographic studies in abdominal exercises: a literature synthesis. J Manipulative Physiol Ther. 2009;32(3):232-44.
ENDEREÇO
Frederico Balbino Lizardo
Instituto de Ciências Biomédicas
Universidade Federal de Uberlândia
Laboratório de Eletromiografia Cinesiológica
Av. Pará, 1720 - Bloco 2A - Sala 2Ao6 - Jardim Umuarama
Submetido: 10/12/2015
Revisado: 26/04/2016
Aceito: 23/05/2016
38400-902 - Uberlândia - MG - BRASIL
e-mail: frederico@ufu.br;
fredbalbinoanato@gmail.com 
